\title{
Effect of Member Length Uncertainty and Backlash on Deformation Accuracy for a High-Precision Space Truss Structure*
}

\author{
Yoya Gon ${ }^{\dagger}$ and Nozomu KoGISO
}

Department of Aerospace Engineering, Osaka Prefecture University, Sakai, Osaka 599-8531, Japan

\begin{abstract}
This study proposes an uncertainty estimation method for the nodal displacement of a truss structure. The truss structure has been used for space structures that require high accuracy, so it is necessary to consider the effects of minute physical uncertainties of the structures. This study considers two types of physical uncertainties: member length uncertainty and position uncertainty due to backlash at the connecting nodes. A structure model is created using a space deployable structure as an example of a truss structure that requires high shape accuracy. Shape accuracy is evaluated using the distortion angle, which is defined as the error with respect to the ideal truss deployment direction. In the first part of the study, the distortion angle due to member length uncertainty is estimated by analyzing the equilibrium of the truss structure model with an uncertain member length. The confidence interval of the distortion angle is then clarified to be linearly related to the magnitude of the uncertainty by applying Monte Carlo simulation, where the uncertainty follows an independent normal distribution. An efficient estimation method for the distortion angle is then established followed by the theoretical derivation of the probabilistic distribution of the distortion angle as a Rayleigh distribution. In the second part of the study, the effect of backlash uncertainty at the connecting nodes is investigated. The backlash is modeled using a virtual cable element having a natural length equal to the backlash size. Finally, the allowable uncertainty range to satisfy a required accuracy is estimated by analyzing the proposed distortion angle while considering both types of uncertainty.
\end{abstract}

Key Words: Truss Structure, Uncertainty, Shape Accuracy, Backlash, Confidence Interval

\section{Nomenclature}

A: numerator of $\tan \theta_{x}$

$B$ : denominator of $\tan \theta_{x}$

$\boldsymbol{d}^{e}$ : nodal displacement vector

$\boldsymbol{f}^{e}$ : initial nodal force vector

$\boldsymbol{F}^{e}$ : nodal force vector

$f\left(\theta_{P}\right)$ : probability distribution function of distortion angle $\theta_{P}$

$\boldsymbol{K}^{e}$ : element stiffness matrix

$l$ : nominal length of triangular batten

$N$ : sample size of Monte Carlo simulation

$\boldsymbol{n}_{b}$ : normal vector of the bottom surface

$\boldsymbol{n}_{t}$ : normal vector of the top surface

$n_{t x}: x$-coordinates of $\boldsymbol{n}_{t}$

$n_{t y}: y$-coordinates of $\boldsymbol{n}_{t}$

$n_{t z}: z$-coordinates of $\boldsymbol{n}_{t}$

$X$ : random variable follows a chi-square distribution with two degrees of freedom

$x_{i}: x$-coordinates of the top surface node

$y_{i}: y$-coordinates of the top surface node

$z_{i}: z$-coordinates of the top surface node

$\alpha$ : lower cumulative probability of the Rayleigh distribution

$\theta_{P}$ : distortion angle

(C) 2021 The Japan Society for Aeronautical and Space Sciences *Presented at the 60th JSASS/JSME Structures Conference, August 1-3, 2018, Tokushima, Japan.

Received 3 January 2020; final revision received 15 May 2020; accepted for publication 28 July 2020.

†Corresponding author, sv102014@edu.osakafu-u.ac.jp
$\theta_{P}^{\alpha}: 100 \alpha$-percentile of the Rayleigh distribution

$\theta_{x}$ : angle between the top surface plane and $x$ axes

$\theta_{y}$ : angle between the top surface plane and $y$ axes

$\sigma$ : standard deviation of member length uncertainty

$\sigma_{t}:$ standard deviation of $\tan \theta_{x}$ and $\tan \theta_{y}$

$\sigma_{z}:$ standard deviation of $z_{i}$

Subscripts

$i$ : $i$ th node of the top surface of truss

\section{Introduction}

Truss structures consist of linear members that sustain only an axial force and are structural systems that can be used to construct economical frames with high stiffness and strength, and are used for various structures around us. Recently, truss structures are being used for space structures that require high accuracy, such as the deployable back-truss structure of the large paraboloid antenna on ASTRO-G, ${ }^{1}$ and the deployable optical bench ${ }^{2}$ of a space X-ray telescope on HITOMI (ASTRO-H). ${ }^{3)}$ In future space astronomy missions, accuracy requirements for these truss structures will be strict in order to achieve better results.

As a future mission, a small satellite for X-ray astronomy observation named "FOcusing Relative universe and Cosmic Evolution (FORCE)" is planned. This satellite will require more precise shape accuracy than that of HITOMI. The primary mission of FORCE is to explore each stratum of an unexplored black hole to elucidate the early history of the universe. For this purpose, the mission will observe an active galactic nucleus via hard X-ray imaging spectroscopy with more than $10 \mathrm{keV}$ power and more than 10 times the spatial 
resolution of conventional observation. ${ }^{4,5)}$ The pointing accuracy requirement for its X-ray telescope, which will have a length of more than $10 \mathrm{~m}$ and include an optical bench built using a truss structure, will be extremely high: 5 arcsec for the attitude determination requirement. In order to achieve the required high accuracy, it is necessary to consider the effects of uncertainty that do not need to be considered on the ground, such as the manufacturing error of the member length and the backlash at the connecting node of the truss structure.

Studies on the uncertainty of member length and backlash have mainly been conducted in the field of dynamic link mechanisms. Several attempts have been made to analyze and synthesize the mechanical uncertainty of function generating linkages. Hartenberg and Denavit ${ }^{6)}$ estimated the maximum output error based on the maximum allowable uncertainty of the member lengths. Kolhatker and Yajnik ${ }^{7)}$ evaluated the maximum output error due to the backlash based on the worst-case analysis of individual backlash. Garrett et al. ${ }^{8)}$ and Dhande et al. ${ }^{9)}$ offered a statistical approach to mechanical uncertainty analysis suitable for both analyzing and synthesizing mechanical uncertainty. Dubowsky and Gardner ${ }^{10)}$ observed that backlash in the joints results in amplification of the internal dynamic system's forces. Du et al. ${ }^{11)}$ developed methods to quantify robustness considering the member length uncertainty.

Regarding truss structures, research on these uncertainties has recently been conducted in the field of space structures that require high shape accuracy. Bauer and Latalski ${ }^{12)}$ formulated an optimal design problem for truss structures considering the member length tolerance. Tzou and Rong ${ }^{13)}$ developed a mathematical model for a three-dimensional spherical joint based on contact force analysis, which includes the effects of normal contact and friction contact. Akita et al. ${ }^{14)}$ proposed a high-precision finite element model and investigated the shape repeatability of pin-jointed bars with frictional contact conditions. Some other studies have been conducted to apply experimental hysteresis to mathematical models in order to establish a nonlinear finite element model of a truss with backlashes. ${ }^{15-19)}$ However, few studies have investigated how these uncertainties propagate to the shape accuracy of a truss structure.

In addition, as a study to reduce the structural error due to these uncertainties, kinematic coupling is utilized at the connection point between the optical bench and the satellite body to release the distortion generated due to thermal deformation, ${ }^{20)}$ or attitude control system of the entire optical bench using actuators is utilized. ${ }^{21,22)}$ However, the amount of the released error is limited. Therefore, it is necessary to clarify the effect of each uncertainty on the shape accuracy of the entire truss structure.

In this study, the effect of these uncertainties on the nodal displacement of a 5-stage truss structure is investigated using numerical simulation. A structure model is created while referencing the deployable optical bench as an example of a truss structure that requires high shape accuracy. The shape accuracy is evaluated by applying the distortion angle, which is defined as the error with respect to the ideal truss deployment direction.

The variation in the distortion angle due to the member length uncertainty is first investigated using a truss structure model, which is created while giving consideration to a space deployable structure, with a member length uncertainty that is assumed to follow an independent normal distribution or an independent uniform distribution. The distortion angle is evaluated by applying Monte Carlo simulation, where the variation in it due to the member length uncertainty is evaluated using the non-linear finite element method.

At first, the effect of the stage size on the distortion angle is investigated, and a 5-stage truss is clarified to be sufficient for investigation. Then, the confidence interval of the distortion angle is obtained and found to be linearly related to the member length uncertainty. In addition, the probabilistic distribution of the distortion angle is theoretically derived to follow the Rayleigh distribution.

Second, the effect of the uncertainty due to the backlash at the connecting nodes is investigated. The backlash is modeled using a virtual cable element having a natural length equal to the backlash size. Then, the allowable initial imperfection size to satisfy the required accuracy is estimated by analyzing the proposed distortion angle considering both the member length uncertainty and the backlash at the connecting nodes.

This paper is organized as follows. In Section 2, details of the truss structure model are described using the structural analysis conditions. Section 3 presents the investigation results focusing on the member length uncertainty. Section 4 discusses the results obtained when modeling the backlash at the connecting nodes and investigating the effects of backlash on the distortion angle. Finally, Section 5 presents our conclusions.

\section{Analysis Truss Structure Model}

\subsection{Analysis model}

The 5-stage truss structure model is shown in Fig. 1; it consists of 63 members and 18 nodes. Each stage is composed of three main components: triangular battens, longerons, and diagonal rods; the latter are tension members with variable lengths that cannot support a compression load, such as a cable. The material properties of the members are listed in Table 1. The longerons and battens are made of aluminum cylinders $10-\mathrm{mm}$ in diameter and 1-mm thick. Their nominal lengths are defined as $380 \mathrm{~mm}$, obtained by referencing the experimental model of previous research. ${ }^{21)}$ The diagonal rods are composed of aluminum cables with a diameter of $2 \mathrm{~mm}$, and are assumed to support only the tension force. Thus, the stiffness is set as zero for the compression load.

\subsection{Shape accuracy analysis}

Even if there is no external force several uncertainties exist, such as member length and the backlash at the nodes generating nodal displacement, which can reduce the shape accuracy of the truss structure. A distorted state can be obtained as a result of the equilibrium conditions. Therefore, 


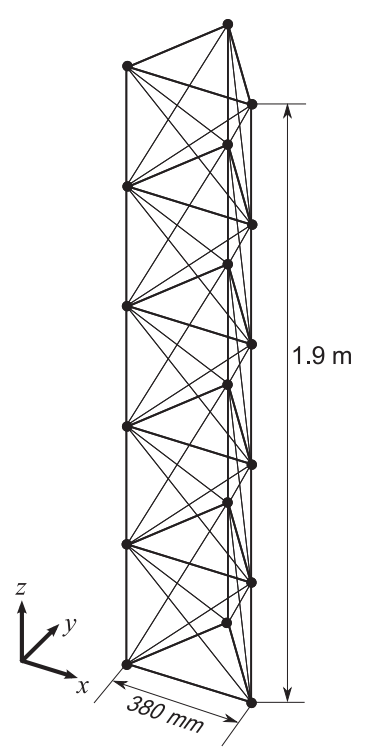

Fig. 1. The analysis truss structure model.

Table 1. Member specifications of the truss structure model for deformation analysis, where all members are made of $\mathrm{Al}$ alloy.

\begin{tabular}{lcccc}
\hline & Triangular & Longeron & \multicolumn{2}{c}{ Diagonal rod } \\
\cline { 4 - 6 } & batten & & Tension & Compression \\
\hline Natural length [mm] & 380 & 380 & 537.4 & 537.4 \\
Cross-sectional area $\left[\mathrm{mm}^{2}\right]$ & 14.9 & 14.9 & 3.1 & 3.1 \\
Young's modulus [GPa] & 70 & 70 & 70 & 0 \\
\hline
\end{tabular}

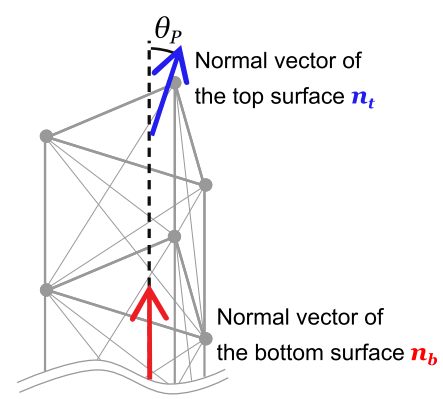

Fig. 2. Definition of the distortion angle, $\theta_{P}$.

the node coordinates of the truss structure model, as shown in Fig. 1, considering the member length uncertainty are calculated using the non-linear finite element method. The member length imperfection imposes an internal force on the truss member. The equilibrium equation is described as follows: ${ }^{23)}$

$$
\boldsymbol{F}^{e}+\boldsymbol{f}^{e}=\boldsymbol{K}^{e} \boldsymbol{d}^{e}
$$

where $\boldsymbol{F}^{e}$ is the nodal force vector, $\boldsymbol{K}^{e}$ is the element stiffness matrix, $\boldsymbol{d}^{e}$ is the nodal displacement vector, and $\boldsymbol{f}^{e}$ is the initial nodal force vector. The initial nodal force is evaluated as the resultant force of all of the initial axial forces resulting from the length imperfection. This equation is solved using Newton's method. ${ }^{24)}$ Then, the shape accuracy is evaluated using the distortion angle, $\theta_{P}$, defined as the angle formed by $\boldsymbol{n}_{b}$, the normal vector of the bottom surface of the truss, and $\boldsymbol{n}_{t}$, the normal vector of the top surface, as shown in

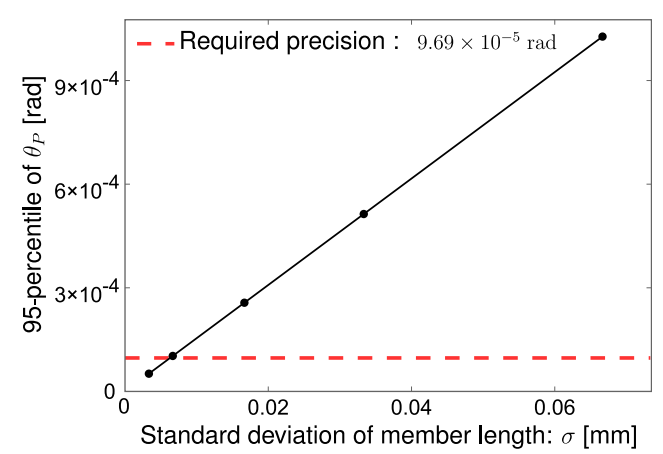

Fig. 3. Relationship of the 95-percentile values of the distortion angle in terms of the standard deviation of member length.

Fig. 2. This distortion angle is evaluated applying the following equation:

$$
\theta_{P}=\cos ^{-1} \frac{\boldsymbol{n}_{t} \cdot \boldsymbol{n}_{b}}{\left|\boldsymbol{n}_{t}\right|\left|\boldsymbol{n}_{b}\right|}
$$

In this study, the target shape accuracy is assumed as $\theta_{P} \leq 9.69 \times 10^{-5} \operatorname{rad}(20 \operatorname{arcsec})$.

\section{Deformation Analysis Based on Member Length Uncertainty}

\subsection{Estimation of allowable interval of uncertainty}

The effect of member length uncertainty on the distortion angle is estimated by applying Monte Carlo simulation under the assumption that the member length uncertainty follows a normal distribution with a zero mean and a standard deviation $\sigma$. The following five patterns of member length uncertainty are considered for the longeron and batten: $\sigma=0.0033 \mathrm{~mm}$, $0.0067 \mathrm{~mm}, 0.017 \mathrm{~mm}, 0.033 \mathrm{~mm}$, and $0.067 \mathrm{~mm}$. The values are set such that the $3 \sigma$ values correspond to member length tolerances of $0.01 \mathrm{~mm}, 0.02 \mathrm{~mm}, 0.05 \mathrm{~mm}, 0.1 \mathrm{~mm}$, and $0.2 \mathrm{~mm}$, respectively. ${ }^{25}$ ) The probability distribution of the distortion angle, $\theta_{P}$, is estimated by applying Monte Carlo simulation with 50,000 samples. Then, the 95-percentile value of $\theta_{P}$ is evaluated as the confidence interval. ${ }^{26)}$

The relationship of the estimated 95-percentile values of $\theta_{P}$ in terms of the member length standard deviation is plotted in Fig. 3. As indicated by the straight line passing through all points, the 95-percentile value represents the liner function in terms of the standard deviation of the member length uncertainty. It is found that the allowable standard deviation for the 95-percentile value is $\sigma=6.30 \times 10^{-3} \mathrm{~mm}$ for the required precision of $9.69 \times 10^{-5} \mathrm{rad}(20 \mathrm{arcsec})$. To confirm the linearity, the 95-percentile value is evaluated by applying Monte Carlo simulation with 50,000 samples for setting the standard deviation of the member length uncertainty as this value. The obtained histogram of $\theta_{P}$ is shown in Fig. 4, where the 95-percentile value of the distortion angle is equal to the estimated value shown above.

\subsection{Estimation of probabilistic distribution of distor- tion angle}

In the above section, the numerical evaluation of the 95percentile value of the distortion angle is discussed. In this 


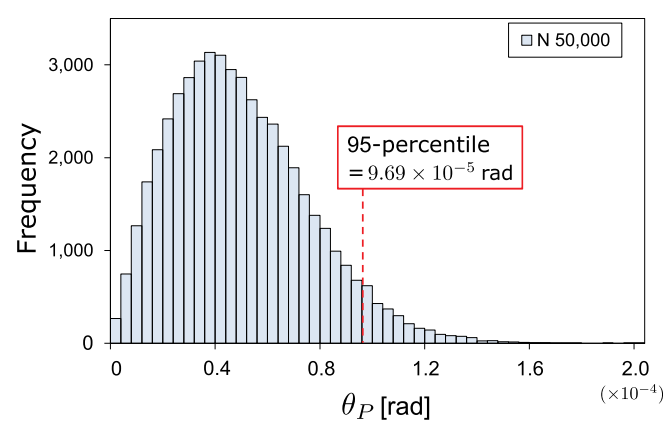

Fig. 4. Histogram of the distortion angle obtained by applying Monte Carlo simulation with 50,000 samples $\left(\sigma=6.30 \times 10^{-3} \mathrm{~mm}\right)$.

section, the probabilistic distribution of $\theta_{P}$ is theoretically derived. Initially, the normal vector of the top surface of the truss, $\boldsymbol{n}_{t}$, is defined as follows:

$$
\boldsymbol{n}_{t}=\left(n_{t x}, n_{t y}, n_{t z}\right)
$$

In comparison, the normal vector of the bottom surface is $\boldsymbol{n}_{b}=(0,0,1)$. Substituting these components into Eq. (2), the distortion angle is obtained as follows:

$$
\theta_{P}=\cos ^{-1} \frac{\boldsymbol{n}_{t} \cdot \boldsymbol{n}_{b}}{\left|\boldsymbol{n}_{t}\right|\left|\boldsymbol{n}_{b}\right|}=\cos ^{-1}\left(\frac{n_{t z}}{\sqrt{n_{t x}^{2}+n_{t y}^{2}+n_{t z}^{2}}}\right)
$$

Then, the plane of the top surface is described as follows:

$$
n_{t x} x+n_{t y} y+n_{t z} z=0
$$

The angles between the top surface plane and $x$ and $y$ axes denoted as $\theta_{x}$ and $\theta_{y}$, respectively, are described as follows:

$$
\tan \theta_{x}=-\frac{n_{t x}}{n_{t z}}, \quad \tan \theta_{y}=-\frac{n_{t y}}{n_{t z}}
$$

Substituting this equation into Eq. (4), $\theta_{P}$ is described using $\theta_{x}$ and $\theta_{y}$ as follows:

$$
\theta_{P}=\cos ^{-1}\left(\frac{1}{\sqrt{\tan ^{2} \theta_{x}+\tan ^{2} \theta_{y}+1}}\right)
$$

Then, the probabilistic distributions of $\tan \theta_{x}$ and $\tan \theta_{y}$ are derived. The nodes of the top surface form an equilateral triangle with length $l$ and are tentatively named 1, 2 and, 3, as shown in Fig. 5. The coordinates of each node are described as $\left(x_{i}, y_{i}, z_{i}\right),(i=1,2,3)$. Both points 1 and 2 are ideally on the $x$-axis.

The values of $\tan \theta_{x}$ and $\tan \theta_{y}$ can be represented as follows:

$$
\begin{aligned}
\tan \theta_{x} & =\frac{\left(y_{3}-y_{1}\right)\left(z_{2}-z_{1}\right)-\left(y_{2}-y_{1}\right)\left(z_{3}-z_{1}\right)}{\left(x_{2}-x_{1}\right)\left(y_{3}-y_{1}\right)-\left(x_{3}-x_{1}\right)\left(y_{2}-y_{1}\right)} \\
\tan \theta_{y} & =\frac{\left(z_{3}-z_{1}\right)\left(x_{2}-x_{1}\right)-\left(z_{2}-z_{1}\right)\left(x_{3}-x_{1}\right)}{\left(x_{2}-x_{1}\right)\left(y_{3}-y_{1}\right)-\left(x_{3}-x_{1}\right)\left(y_{2}-y_{1}\right)}
\end{aligned}
$$

In this model, the following relationships are satisfied: $y_{1} \simeq y_{2}, \quad x_{2}-x_{1} \simeq l, \quad x_{3}-x_{1} \simeq \frac{l}{2}, \quad y_{3}-y_{1} \simeq \frac{\sqrt{3}}{2} l$

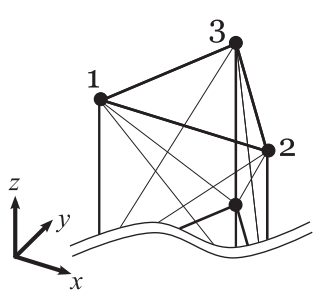

Fig. 5. Node ID of the top surface of the three nodes as vertices of an equilateral triangle.

The reason to hold Eq. (10) is shown below. For example, the sensitivities of $\tan \theta_{x}$ with respect to $x_{i}, y_{i}$, and $z_{i}(i=2,3)$ can be represented as follows:

$$
\begin{aligned}
\frac{\partial}{\partial x_{2}} \tan \theta_{x} & =-\left(y_{3}-y_{1}\right) \frac{B}{A^{2}} \\
\frac{\partial}{\partial y_{2}} \tan \theta_{x} & =\left(x_{3}-x_{1}\right) \frac{B}{A^{2}}-\left(z_{3}-z_{1}\right) \frac{1}{A} \\
\frac{\partial}{\partial z_{2}} \tan \theta_{x} & =\left(y_{3}-y_{1}\right) \frac{1}{A} \\
\frac{\partial}{\partial x_{3}} \tan \theta_{x} & =\left(y_{2}-y_{1}\right) \frac{B}{A^{2}} \\
\frac{\partial}{\partial y_{3}} \tan \theta_{x} & =-\left(x_{2}-x_{1}\right) \frac{B}{A^{2}}+\left(z_{2}-z_{1}\right) \frac{1}{A} \\
\frac{\partial}{\partial z_{3}} \tan \theta_{x} & =-\left(y_{2}-y_{1}\right) \frac{1}{A}
\end{aligned}
$$

where $A$ and $B$ denote the numerator and denominator in Eq. (8), respectively. When $\theta_{x}$ is small, $A \gg B$ holds. In addition, if there is no uncertainty on the member length, the following equations, $y_{1}=y_{2}, y_{3}-y_{1}=(\sqrt{3} / 2) l$, and $z_{1}=z_{2}=z_{3}$ hold. Even if uncertainty of the member length is considered, the following equations, $y_{3}-y_{1} \gg y_{2}-y_{1}$, $y_{3}-y_{1} \gg z_{2}-z_{1}$, and $y_{3}-y_{1} \gg z_{3}-z_{1}$ hold, because variations of $x, y$ and $z$-coordinate values are much smaller than the nominal member length $l$. Therefore, the sensitivity of $\tan \theta_{x}$ with respect to $z_{2}$ is larger than the other terms. Similarly, the sensitivities of $\tan \theta_{y}$ with respect to $z_{2}$ and $z_{3}$ are larger than the other terms.

Substituting Eq. (10) into Eqs. (8) and (9), the following equations are obtained:

$$
\begin{aligned}
\tan \theta_{x} & =\frac{1}{l}\left(z_{2}-z_{1}\right) \\
\tan \theta_{y} & =\frac{1}{\sqrt{3} l}\left(z_{1}+z_{2}-2 z_{3}\right)
\end{aligned}
$$

The variation in the $z$-coordinate value of each node on the top surface is nearly equal to the summation of the member length uncertainties of the longerons under the nodes. When the member length uncertainty follows an independent normal distribution, the variation in the $z$-coordinate of each node on the top surface can be modeled to follow a normal distribution as $N\left(\mu_{z_{i}}, \sigma_{z_{i}}^{2}\right),(i=1,2,3)$. Because $\tan \theta_{x}$ and $\tan \theta_{y}$ are described as linear functions of the $z$-coordinates, as described in Eqs. (17) and (18), they also follow a normal distribution as follows: 


$$
\begin{aligned}
& \tan \theta_{x}=\frac{1}{l}\left(z_{2}-z_{1}\right) \sim N\left(0, \frac{2 \sigma_{z}^{2}}{l^{2}}\right) \\
& \tan \theta_{y}=\frac{1}{\sqrt{3} l}\left(z_{1}+z_{2}-2 z_{3}\right) \sim N\left(0, \frac{2 \sigma_{z}^{2}}{l^{2}}\right)
\end{aligned}
$$

It should be noted that $\tan \theta_{x}$ and $\tan \theta_{y}$ follow an identical normal distribution. The standard deviation of $\tan \theta_{x}$ and $\tan \theta_{y}$ is denoted as $\sigma_{t}$.

For example, consider the case of $\sigma=6.30 \times 10^{-3} \mathrm{~mm}$ as the allowable standard deviation, as described in Section 3.1. In this case, $\sigma_{t}$ yields $3.96 \times 10^{-5}$. For confirmation, distributions of $\tan \theta_{x}$ and $\tan \theta_{y}$ are evaluated by applying Monte Carlo simulation using 50,000 samples. The histograms obtained for $\tan \theta_{x}$ and $\tan \theta_{y}$ are shown in Fig. 6. The red curve indicates the probabilistic density of $N\left(0, \sigma_{t}^{2}\right)$. This suggests that $\tan \theta_{x}$ and $\tan \theta_{y}$ follow the same normal distribution.

Here, the following random variable is introduced:

$$
X=\frac{\left(\tan \theta_{x}\right)^{2}+\left(\tan \theta_{y}\right)^{2}}{\sigma_{t}^{2}}
$$

where $X$ follows a chi-square distribution with two degrees of freedom. ${ }^{27)}$ From Eqs. (7) and (21), the following equation is obtained:

$$
\begin{aligned}
\theta_{P} & =\cos ^{-1}\left(\frac{1}{\sqrt{\tan ^{2} \theta_{x}+\tan ^{2} \theta_{y}+1}}\right) \\
& =\cos ^{-1}\left(\frac{1}{\sqrt{\sigma_{t}^{2} X+1}}\right)
\end{aligned}
$$

Then, $X$ and $\mathrm{d} X / \mathrm{d} \theta_{P}$ are evaluated as follows:

$$
\begin{aligned}
X & =\frac{1}{\sigma_{t}^{2}}\left(\frac{1}{\cos ^{2} \theta_{P}}-1\right)=\frac{\tan ^{2} \theta_{P}}{\sigma_{t}^{2}} \\
\frac{\mathrm{d} X}{\mathrm{~d} \theta_{P}} & =\frac{1}{\sigma_{t}^{2}}\left(\frac{2 \tan \theta_{P}}{\cos ^{2} \theta_{P}}\right)=\frac{2 \tan \theta_{P}\left(1+\tan ^{2} \theta_{P}\right)}{\sigma_{t}^{2}}
\end{aligned}
$$

Using these equations, the probabilistic density function of $\theta_{P}$ can be described as follows: ${ }^{28)}$

$$
\begin{array}{r}
f\left(\theta_{P}\right)=f_{X}(x)\left|\frac{\mathrm{d} x}{\mathrm{~d} \theta_{P}}\right| \\
=\frac{\tan \theta_{P}\left(1+\tan ^{2} \theta_{P}\right)}{\sigma_{t}^{2}} \exp \left[-\frac{\tan ^{2} \theta_{P}}{2 \sigma_{t}^{2}}\right] \\
\left(\because 0 \leq \theta_{P} \leq \frac{\pi}{2}\right)
\end{array}
$$

When $\theta_{P}$ is small, the following equations for $\tan \theta_{P} \approx \theta_{P}$ and $1+\theta_{P}^{2} \approx 1$ hold. Then, the probabilistic density function can be approximated as follows:

$$
f\left(\theta_{P}\right)=\frac{\theta_{P}}{\sigma_{t}^{2}} \exp \left[-\frac{\theta_{P}^{2}}{2 \sigma_{t}^{2}}\right]
$$

Thus, $\theta_{P}$ follows a Rayleigh distribution with parameter $\sigma_{t}$.

The histogram of $\theta_{P}$, as shown in Fig. 4, and Rayleigh distribution with parameter $\sigma_{t}=3.96 \times 10^{-5}$ are compared in

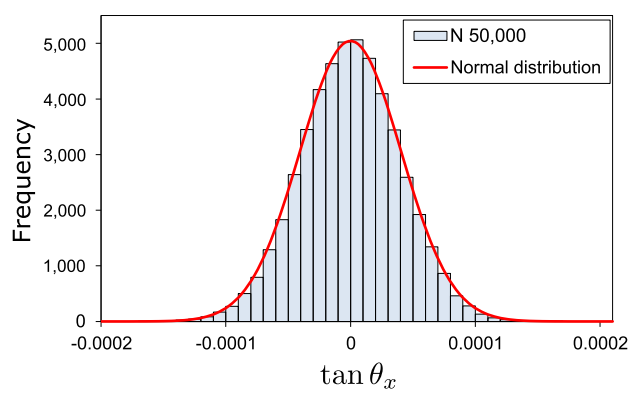

(a) Histogram of $\tan \theta_{x}$

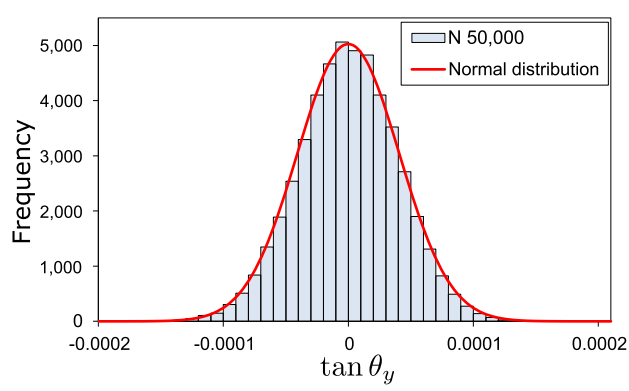

(b) Histogram of $\tan \theta_{y}$

Fig. 6. Comparison of the estimated normal distribution $\left(\mu=0, \sigma_{t}=\right.$ $\left.3.96 \times 10^{-5}\right)$ and histograms of $\tan \theta_{x}$ and $\tan \theta_{y}\left(\sigma=6.30 \times 10^{-3} \mathrm{~mm}\right)$.

Fig. 7. It is found that the distribution of the distortion angle agrees with the probability density function of the Rayleigh distribution illustrated as a red solid curve. In addition, the lower cumulative probability, $100 \alpha \%$, of the Rayleigh distribution at $\theta_{P}=\theta_{P}^{\alpha}$ and the $100 \alpha$-percentile value, $\theta_{P}^{\alpha}$, of the Rayleigh distribution are obtained as follows: ${ }^{29)}$

$$
\begin{aligned}
\alpha & =\int_{0}^{\theta_{P}^{\alpha}} f\left(\theta_{P}\right) d \theta_{P}=1-\exp \left[-\frac{\theta_{P}^{\alpha 2}}{2 \sigma_{t}^{2}}\right] \\
\theta_{P}^{\alpha} & =\sqrt{-2 \sigma_{t}^{2} \ln (1-\alpha)}
\end{aligned}
$$

When $\sigma_{t}=3.96 \times 10^{-5}$ and $\alpha=0.95$ are substituted into Eqs. (27) and (28), $\theta_{P}^{\alpha}=9.69 \times 10^{-5} \mathrm{rad}$ is obtained. This value is the same as that obtained through Monte Carlo simulation, as shown in Fig. 4. Specifically, the uncertainty of the distortion angle follows a Rayleigh distribution when the member length uncertainty follows a normal distribution.

\subsection{Effect of probabilistic distribution}

The above results, including those of the distortion angle, follow the Rayleigh distribution obtained under the assumption that the member length follows a normal distribution. In this section, the effect of other probabilistic distributions is investigated by considering a uniform distribution with $[-0.2,0.2] \mathrm{mm}$. The histograms obtained by applying Monte Carlo simulation performed with 5,000 samples are compared in Fig. 8, where 1-stage models with normal and uniform distributions and a 30-stage model are compared. On each graph, the Rayleigh distributions estimated for each case are overlapped as a red curve. As a result of the goodness-of-fit test ${ }^{27)}$ for each model, it was concluded that the 1-stage model with uniform distribution does not follow the Rayleigh distribution, which is in contrast to the 30-stage model. It is assumed that the distribution type effect becomes 


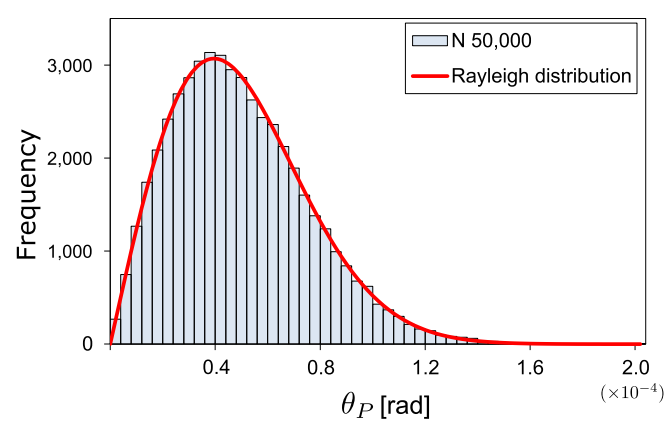

Fig. 7. Comparison of the estimated Rayleigh distribution $\left(\sigma_{t}=\right.$ $\left.3.96 \times 10^{-5}\right)$ and histogram of the $\theta_{P}$ uncertainty $\left(\sigma=6.30 \times 10^{-3} \mathrm{~mm}\right)$.

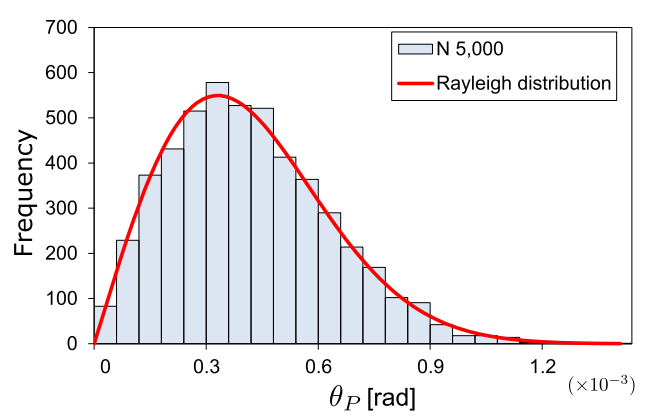

(a) 1-stage model under a normal distribution

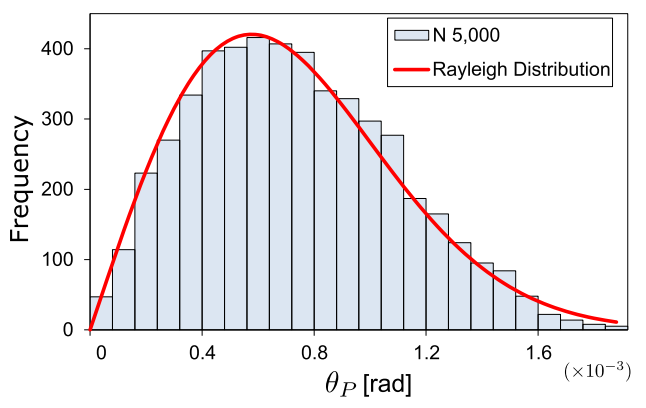

(b) 1-stage model under a uniform distribution

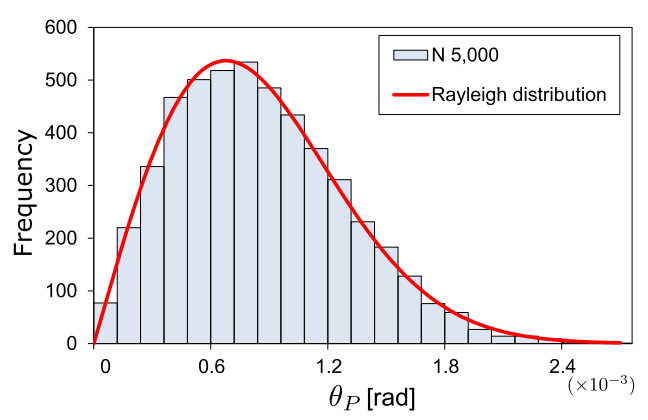

(c) 30-stage model under a uniform distribution

Fig. 8. Comparison of the histograms obtained by applying Monte Carlo simulation with 5,000 samples and derived Rayleigh distribution.

negligible as the number of stages increases as a result of the central limit theorem.

\subsection{Effect of the number of stages}

As the next step, the effect of the number of stages in a truss structure model has on the distortion angle is investigated. The 95-percentile values of the distortion angle for several numbers of stages are evaluated by applying Monte Carlo simulation with 5,000 samples for $3 \sigma=0.2 \mathrm{~mm}$.

The 95-percentile values of the distortion angle, $\theta_{P}$, for

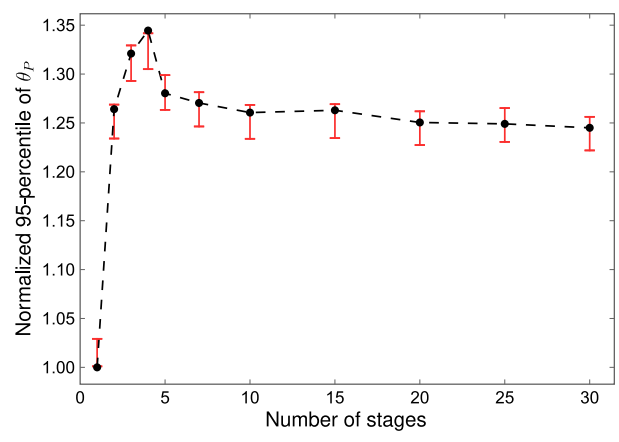

Fig. 9. Relationship of the normalized 95-percentile value of $\theta_{P}$ in terms of number of stages and $95 \%$ confidence interval.

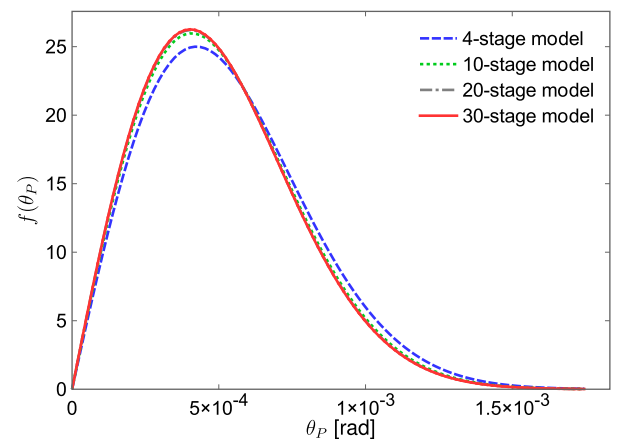

Fig. 10. The probability density functions of Rayleigh distribution corresponding to the histograms of $\theta_{P}$ in the $4,10,20$, and 30-stage models in the case of $3 \sigma=0.2 \mathrm{~mm}$.

each number of stages are shown in Fig. 9, and then the values are normalized to those of the 1-stage model. The error bars of the red line indicate $95 \%$ confidence intervals of the 95-percentile value of $\theta_{P}$. It is found that the 95\% confidence level for the different number of stages in the models does not change very much for a constant sample size. Therefore, the sample size is sufficient to evaluate the 95-percentile value of $\theta_{P}$. The value peaks for the 4-stage model and then decreases as the number of stages increases. However, the value is practically converged to a constant value. For example, the probability density functions of Rayleigh distribution corresponding to the histograms of $\theta_{P}$ in the $4,10,20$, and 30 -stage models in the case of $3 \sigma=0.2 \mathrm{~mm}$ are compared in Fig. 10. The variance of the 30-stage model is smaller than that of the 4-stage model, and the $f\left(\theta_{P}\right)$ converges to a constant as the number of the stages increases. It is assumed that the member length uncertainty will cancel out the effect on $\theta_{P}$ from each other and will converge to some constant value when the number of stages increases. It is found that the 5stage truss is clarified to be sufficient for investigation, even if the results for a structure with more stages is desired.

\section{Deformation Analysis considering Backlash Uncer- tainty}

\subsection{Backlash model}

A truss structure has a pin joint, and there is backlash between the hole and the shaft. This causes uncertainty in the node position. For this study, the magnitude of the backlash uncertainty is assumed to be about $0.01 \mathrm{~mm}$ in each node. ${ }^{30)}$ 


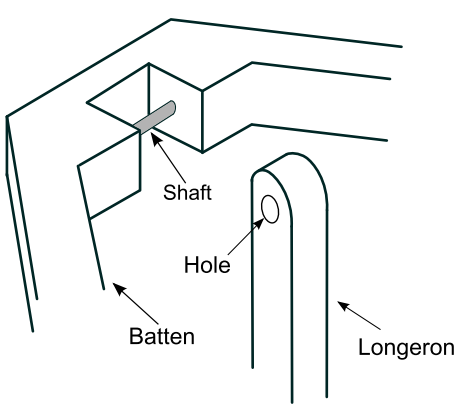

(a) Backlash caused by a hole of the longeron and the shaft of the batten at the connecting node

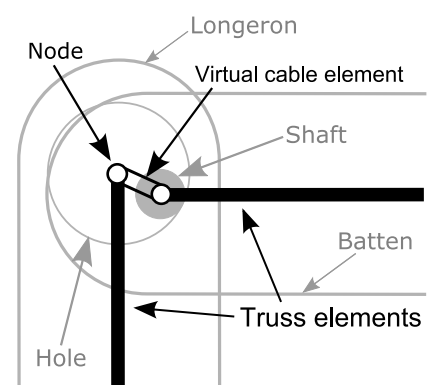

(b) Virtual cable clement describing the backlash uncertainty for the structural analysis

Fig. 11. Backlash uncertainty at a connecting node.

In comparison to the allowable member length uncertainty of $\sigma=6.30 \times 10^{-3} \mathrm{~mm}$, the backlash uncertainty cannot be ignored.

It is assumed that a batten is connected to a longeron at the node by fitting a shaft of the batten into a hole of the longeron as shown in Fig. 11(a). The backlash is modeled by introducing a virtual cable element connecting the ends of the batten and longeron, as shown in Fig. 11(b), where both of the end nodes are located at the centers of the shaft and hole, respectively. The length of the cable element is equal to the difference in the diameters of the hole and shaft. To model the backlash, the stiffness of the cable element is set as infinite on the tensile side and zero on the compression side. The backlash model in this study considers only backlash in the direction perpendicular to the shaft.

\subsection{Shape accuracy considering the backlash}

\subsubsection{Analysis of two-dimensional model with backlash}

The structural analysis of a simple two-dimensional truss model with backlash, as shown in Fig. 12, is performed to confirm the validity of the backlash model with virtual cable elements. The truss is composed of four truss members on each side $380-\mathrm{mm}$ long and two diagonal rods. The four corners are assumed to have backlash as large as $30 \mathrm{~mm}$, which is extremely large to illustrate the validity when backlash is modeled using virtual cable elements.

To eliminate the backlash by applying some tension force to the diagonal rod, the diagonal rods are shortened from $537.4 \mathrm{~mm}$ to $445.1 \mathrm{~mm}$. Then, the equilibrium state is evaluated applying structural analysis with the two lower batten nodes fixed. The tension stiffness of the virtual cable element is set to $10^{5}$ times that of the truss members, and the compression is fixed as $10^{-5}$ times that of the tension stiffness.

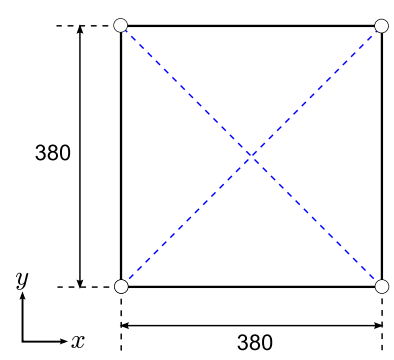

Fig. 12. Two-dimensional truss structure model.

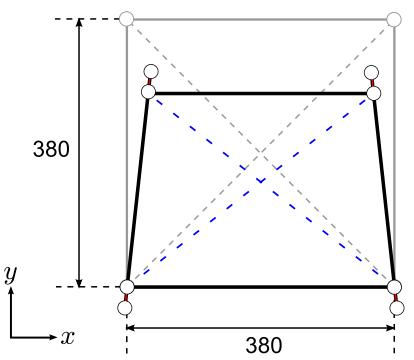

(a) Equilibrium state

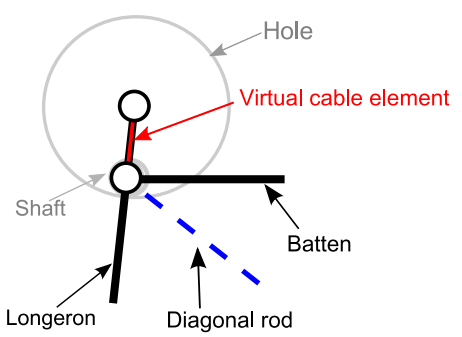

(b) Enlarged view around the upper left node

Fig. 13. Two-dimensional truss structure model with backlash (i.e., diagonal rod length shortened to $445.1 \mathrm{~mm}$ ).

The equilibrium state is shown in Fig. 13(a), and the enlarged view at the upper left node is shown in Fig. 13(b). It is found that the total height of the truss is shortened by changing the length of the diagonal rod. Then, the shaft at the end of the batten contacts the edge of the hole that is located in the axial direction of the longeron. That is, modeling backlash using virtual cable elements is reasonable.

\subsubsection{Analysis of three-dimensional model with back- lash}

The structural analysis of a three-dimensional 1-stage model with backlash, as shown in Fig. 14, is performed to confirm the validity of modeling the backlash with virtual cable elements. The six corners are assumed to have backlash as large as $30 \mathrm{~mm}$, similar to the previous example. To eliminate the backlash by applying some tension force to the diagonal rod, the diagonal rods are shortened from $537.4 \mathrm{~mm}$ to $428.1 \mathrm{~mm}$. Then, the equilibrium state is evaluated by performing a structural analysis in which the three lower batten nodes are fixed and the other analysis conditions are the same as those described in the previous section. In the threedimensional model shown in Fig. 15, the longeron is not tilted when the backlash is considered. The equilibrium state is shown in Fig. 15(a), and an enlarged view of the upper left node is shown in Fig. 15(b). 


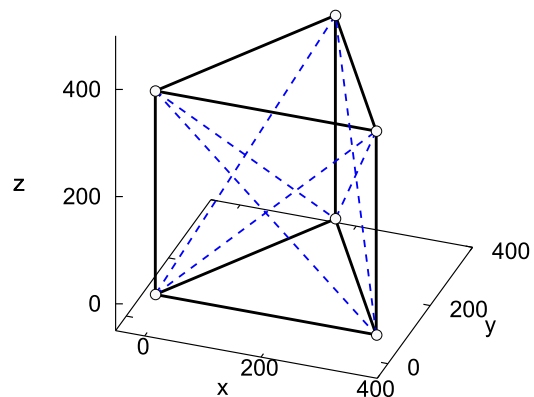

Fig. 14. Three-dimensional 1-stage truss structure model.

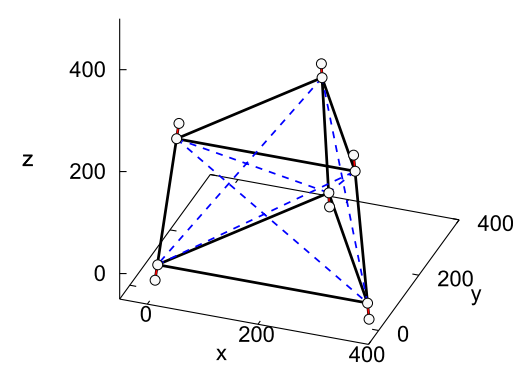

(a) Equilibrium state

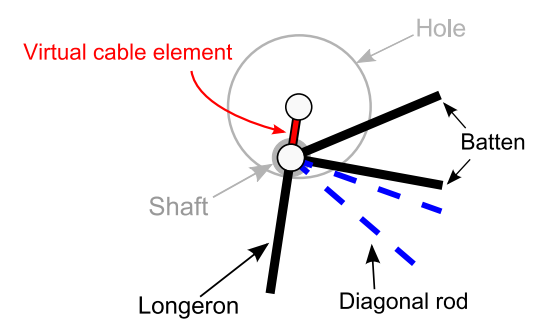

(b) Enlarged view of the region around the upper left node

Fig. 15. Three-dimensional 1-stage truss structure model with backlash (i.e., diagonal rod length shortened to $428.1 \mathrm{~mm}$ ).

As in the case of the two-dimensional model, modeling backlash using a virtual cable element is also found to be reasonable for the three-dimensional model.

\subsubsection{Analysis of 5-stage truss model considering both member length uncertainty and backlash}

The distortion angle, $\theta_{P}$, of the 5-stage truss structure model with backlash at the connecting node between the longeron and batten and the member length imperfection are evaluated. The backlash is modeled by a virtual cable element, and it is assumed that the size of the backlash follows a uniform distribution with [0.004, 0.017] $\mathrm{mm}$ as an actual size. ${ }^{30)}$ Six patterns of the member length uncertainty are considered for the longeron and batten: $\sigma=0.0067 \mathrm{~mm}$, $0.013 \mathrm{~mm}, 0.017 \mathrm{~mm}, 0.033 \mathrm{~mm}, 0.05 \mathrm{~mm}$, and $0.067 \mathrm{~mm}$. The values are set such that the $3 \sigma$ values correspond to a member length tolerance of $0.02 \mathrm{~mm}, 0.04 \mathrm{~mm}, 0.05 \mathrm{~mm}$, $0.1 \mathrm{~mm}, 0.15 \mathrm{~mm}$, and $0.2 \mathrm{~mm}$, respectively. To facilitate the diagonal rods under tensile loads, it is assumed that the length uncertainty of the diagonal rods follows a uniform distribution between $0 \mathrm{~mm}$ and six values as $-0.02 \mathrm{~mm}$, $-0.04 \mathrm{~mm},-0.05 \mathrm{~mm},-0.1 \mathrm{~mm},-0.15 \mathrm{~mm}$, and $-0.2 \mathrm{~mm}$. The negative values are selected to stabilize the node position by applying tensile loads to the diagonal rods. The distortion angle, $\theta_{P}$, is then estimated by a Monte Carlo simulation with

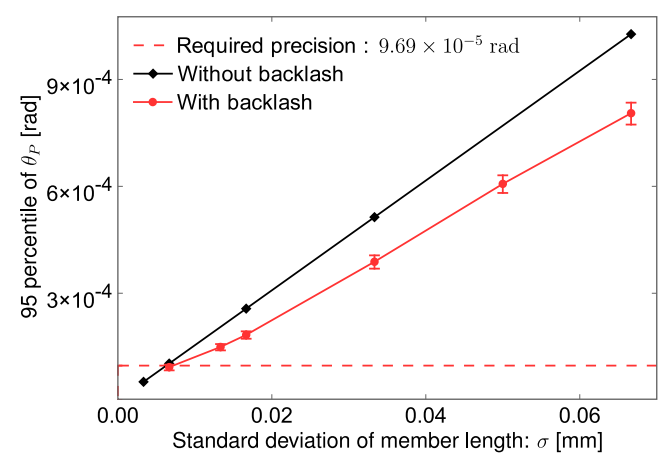

Fig. 16. Relationships of the 95-percentile values of the distortion angle with and without the backlash based on the standard deviation of member length.

1,000 samples, and the 95-percentile value of the distortion angle is evaluated.

The relationships of the 95-percentile values of the distortion angle with and without backlash based on the standard deviation of member length are plotted as red and black lines in Fig. 16, respectively. The black line is the same as shown in Fig. 3. The error bars of the red line indicate 95\% confidence intervals of the 95-percentile value of $\theta_{P}$. They indicate the estimation accuracy related to the sample size, and can be obtained in the same way as described in Section 3.4. It is found that the sample size is sufficient to evaluate the 95-percentile value of $\theta_{P}$. The $95 \%$ confidence intervals of $\sigma_{t}$ while considering the sample size can be obtained using the chi-square distribution.

It is found that the 95-percentile values of $\theta_{P}$ with the backlash become smaller than those without the backlash when member length uncertainty is large. That is, the backlash is believed to partially alleviate the effect of member length uncertainty because the position of the shaft with respect to the hole can move freely as the result of backlash. Even if the member length changes, the node position can be moved by the width of the backlash without generating internal force on members.

The allowable standard deviation for the 95-percentile value with backlash is $\sigma=7.18 \times 10^{-3} \mathrm{~mm}$ for the required precision of $9.69 \times 10^{-5} \mathrm{rad}(20 \mathrm{arcs})$. This value is alleviated from $6.30 \times 10^{-3} \mathrm{~mm}$, the estimated allowable standard deviation without backlash.

On the other hand, the difference of the 95-percentile values becomes smaller as the member length uncertainty is reduced. This indicates that backlash uncertainty has a dominant effect on the 95-percentile value of $\theta_{P}$ when the member length uncertainty is smaller than the backlash uncertainty.

\section{Conclusion}

This study investigates the effect of uncertainties in the member length and backlash on the nodal displacement at the connecting rods of a truss structure by developing numerical evaluation methods. A truss structure model is created referencing the space deployable structure, and the equilibrium state under the uncertainties is evaluated using the non- 
linear finite element method. From the numerical calculations, the following conclusions are drawn:

1. First, uncertainty in the distortion angle is determined by clarifying that the shape accuracy is linearly related to the member length uncertainty, which is assumed to follow independent normal distribution. We then propose a method to estimate the tolerance value of the member length uncertainty that satisfies the required distortion angle.

2. It is found that the distortion angle follows a Rayleigh distribution when member length uncertainty follows a normal distribution. In addition, even when member length uncertainty follows a uniform distribution, the probabilistic distribution of the distortion angle is shown to converge to a Rayleigh distribution using the central limit theorem.

3. A new modeling method is proposed to model the backlash uncertainty at a connecting node by introducing a virtual cable element. The efficiency of the proposed method is demonstrated through numerical examples.

4. Even when both the member length uncertainty and backlash uncertainty are considered, the tolerance value of the member length uncertainty under an appropriate value of the backlash uncertainty can be evaluated. This is because the non-linearity due to the backlash uncertainty in the distortion angle uncertainty is weak.

A future problem is to consider the effect of the friction uncertainty at the connecting nodes on the nodal displacement of a truss structure.

\section{Acknowledgments}

Part of this study was financially supported by the strategic research and development funds of ISAS/JAXA and JSPS KAKENHI 18K18812.

\section{References}

1) Saito, H., Sakai, S., Higuchi, K., Kishimoto, N., Takeuchi, H., Yoshihara, K., Asaki, Y., Tsuboi, M., Murata, Y., and Kobayashi, H.: Advanced Space Technologies in Space Science Missions - Space VLBI Mission ASTRO-G Project as an Example, Trans. JSASS Aerospace Technology Japan, 8, ists27 (2010), pp. To_4_33-To_4_41.

2) Kitamura, T., Natori, M., Yamashiro, K., and Obata, A.: Development of a High Stiffness Extendible and Retractable Mast 'Himat' for Space Applications, AIAA Paper 90-1054-CP, 1990.

3) Takahashi, T., Kokubun, M., Mitsuda, K., Kelley, R., Ogashi, T., Aharonian, F., et al.: The ASTRO-H (Hitomi) X-ray Astronomy Satellite, Proc. SPIE, 9905 (2016), $99050 \mathrm{U}$.

4) Mori, K., Tsuru, T., Nakazawa, K., Ueda, Y., Okajima, T., Murakami, H., Awaki, H., Matsumoto, H., Fukazawa, Y., Tsunemi, H., Takahashi, T., and Zhang, W. W.: Focusing on Relativistic Universe and Cosmic Evolution: the FORCE Mission, Proceedings of the XII Multifrequency Behaviour of High Energy Cosmic Sources Workshop, Palermo, Italy, 306-077, 2017.

5) Focusing on Relativistic Universe and Cosmic Evolution Concept Study Paper, 2016/11/26, http://www.cc.miyazaki-u.ac.jp/force/wpcontent/uploads/force_proposal.pdf, (in Japanese, accessed February 16, 2020).

6) Hartenberg, R. S. and Denavit, J.: Kinematic Synthesis of Linkages, McGraw-Hill, New York, 1964, pp. 316-319.

7) Kolhatker, S. A. and Yajnik, K. S.: The Effects of Play in the Joints of a Function Generating Mechanism, J. Mechanisms, 5, 4 (1970), pp. 521-532.

8) Garrett, R. E. and Hall, A. S., Jr.: Effect of Tolerance and Clearance in
Linkage Design, ASME J. Eng. Ind., 91, 1 (1969), pp. 198-202.

9) Dhande, S. G. and Chakraborty, J.: Analysis and Synthesis of Mechanical Error in Linkages - a Stochastic Approach, ASME J. Eng. Ind., 95, 3 (1973), pp. 672-676.

10) Dubowsky, S. and Gardner, T. N.: Design and Analysis of Multilink Flexible Mechanisms with Multiple Clearance Connections, ASME J. Eng. Ind., 99, 1 (1977), pp. 88-96.

11) Du, X. P., Venigella, P. K., and Liu, D.: Robust Mechanism Synthesis with Random and Interval Variables, Mechanism and Machine Theory, 44, 7 (2009), pp. 1321-1337.

12) Bauer, J. and Latalski, J.: Manufacturing Tolerances of Truss Members' Lengths, Comput. Assist. Mech. Eng. Sci., 7, 4 (2000), pp. 461-469.

13) Tzou, H. S. and Rong, Y.: Contact Dynamics of a Spherical Joint and a Jointed Truss-cell System, AIAA J., 29, 1 (1991), pp. 81-88.

14) Akita, T., Nakajima, K., and Tanaka, H.: A Numerical Study on Shape Repeatability of Pin Jointed Beam with Frictional Contact Conditions, 31st International Symposium on Space Technology and Science, Matsuyama, Japan, 2017-c-33, 2017.

15) Dutson, J. D. and Folkman, S. L.: A Nonlinear Finite Element Model of a Truss using Pinned Joints, AIAA Paper 96-1408, 1996.

16) Folkman, S. L., Roswell, E. A., and Ferney, G. D.: Influence of Pinned Joints on Damping and Dynamic Behavior of a Truss, J. Guid. Control Dynam., 18, 6 (1995), pp. 1398-1403.

17) Okuizumi, N. and Natori, M. C.: Nonlinear Vibrations of a Satellite Truss Structure with Gaps, AIAA Paper 2004-1867, 2004.

18) Hayashi, M., Senba, A., and Matsumoto, T.: Non-linear System Identification of Extensible Truss Structures Using Hilbert Transform, 31st International Symposium on Space Technology and Science, Matsuyama, Japan, 2017-c-48, 2017.

19) Senba, A., Ota, T., and Matsumoto, T.: Non-linear Transfer Characteristics of Truss Structures with Joints for Extensible Mechanism, 32nd International Symposium on Space Technology and Science, Fukui, Japan, 2019-c-21, 2019.

20) Ishimura, K., Tanaka, H., Ogi, Y., Ogawa, Y., Tsunode, H., Sato, Y., Maeda, O., and Abe, K.: Approximated Completion Conditions of Kinematic Couplings for Precise Deployable Structures, 32nd International Symposium on Space Technology and Science, Fukui, Japan, 2019-c-01, 2019.

21) Funakoshi, Y., Ishimura, K., Hagi, Y., and Iwasa, T.: Application of Preview Information to Pointing Control of Truss Structure Using Artificial Thermal Expansion on Orbit, J. Intell. Mater. Syst. Struct., 26, 6 (2015), pp. 730-739.

22) Gallo, L. C., Koujelev, A., Gagnon, S., Elgin, T., Guibert, M., Iizuka, R., Ishida, M., Ishimura, K., Iwata, N., Kawano, T., Lambert, C., Moroso, F., Ueno, S., and Yuasa, T.: In-flight Performance of the Canadian Astro-H Metrology System, J. Astron. Telesc. Instrum. Syst., 4, 2 (2018), 021405.

23) Yang, T. Y.: Finite Element Structural Analysis, Prentice-Hall, Englewood Cliffs, 1986, pp. 83-90.

24) Arora, J. S.: Introduction to Optimum Design, Academic Press, New York, 2011, pp. 459-466.

25) JIS B 0405, General Tolerances, Part 1: Tolerances for Linear and Angular Dimensions without Individual Tolerance Indications, 1991.

26) Piersol, A. G.: Procedures to Compute Maximum Structural Responses from Predictions or Measurements at Selected Points, Shock Vibration, 3, 3 (1996), pp. 211-221.

27) Ang, A. H.-S. and Tang, W. H.: Probability Concepts in Engineering: Emphasis on Applications to Civil and Environmental Engineering, Wiley, New York, 2006, pp. 289-296.

28) Thoft-Christensen, P. and Baker, M. J.: Structural Reliability Theory and Its Applications, Springer, 1982.

29) Minotani, T.: Statistical Distribution Handbook, Asakura Publishing, Tokyo, 2003, pp. 670-674 (in Japanese).

30) JIS B 0401-1, Geometrical Product Specifications (GPS), ISO Code System for Tolerances on Linear Sizes - Part 1: Basis of Tolerances, Deviations and Fits, 2016. 